\title{
Desarrollo y validación de la Escala de Distancia Social hacia Personas Trans
}

\section{Development and Validation of the Social Distance towards Trans Persons Scale}

\author{
Caleb Esteban * 1, Juan Aníbal González-Rivera ${ }^{2}$, Margarita Francia-Martínez ${ }^{3}$, \\ Zahira Lespier ${ }^{4}$ \\ 1 - Ponce Health Sciences University, Ponce, Puerto Rico. \\ 2 - Ponce Health Sciences University, San Juan University Center, Puerto Rico. \\ 3 - Universidad Carlos Albizu, San Juan, Puerto Rico. \\ 4 - Administración de Servicios de Salud Mental y Contra la Adicción, San Juan, Puerto Rico.
}

Introducción

Método

Resultados

Discusión

Referencias

Recibido: 17/01/2019 Revisado: 25/02/2019 Aceptado: 10/03/2019

\section{Resumen}

El presente estudio analiza las propiedades psicométricas de la Escala de Distancia Social hacia Personas Trans (DS-T) en una muestra de profesionales y estudiantes graduados en psicología. Además, se analizó si existen diferencias significativas en la distancia social hacia la comunidad trans entre estudiantes y profesionales de la psicología. La muestra contó con 127 participantes reclutados por disponibilidad. Los resultados confirman que la escala posee una estructura unidimensional. Los 13 ítems desarrollados cumplieron con los criterios de discriminación y obtuvieron cargas factoriales apropiadas. El índice alfa de Cronbach de la DS-T fue de .99. No se encontraron diferencias significativas en la distancia social hacia personas trans entre estudiantes y profesionales de la psicología. Se determinó cómo la distancia y la cercanía constituyen extremos de la empatía, por lo que cuanto mayor sea la distancia hacia una persona o comunidad, menor será la probabilidad de desarrollar empatía hacia estas personas.

Palabras clave: distancia social, transgénero, transexual, género, validación

\begin{abstract}
The present study analyzes the psychometric properties of the Social Distance Scale toward Trans Persons (DS-T) in a sample of psychology professionals and graduate students. In addition, it analyzes whether there are significant differences in the social distance to the trans community between psychology students and professionals. The sample had 127 participants, who were recruited based on their availability. The results confirm that the scale has a one-dimensional structure. The 13 items developed by the researchers met the criteria of discrimination and showed appropriate factor loads. The Cronbach alpha internal consistency index for DS-T was .99. There were no significant differences in social distance towards trans people between psychology students and professionals. It was determined how distance and closeness constitute the extremes of empathy, so the larger the distance from a person or community, the lower the probability of being empathetic toward them.
\end{abstract}

Keywords: social distance, transgender, transsexual, gender, validation

\footnotetext{
* Correspondencia a: Dr. Caleb Esteban, Ph.D., Clinical Psychology Program, School of Behavioral and Brain Sciences, Ponce Health Sciences University, PO BOX 7004, Ponce, Puerto Rico 00732-7004. Email: cesteban@psm.edu. ORCID: https://orcid.org/0000-0002-0960-6311

Cómo citar este artículo: Esteban, C., González-Rivera, J. A., Francia-Martínez, M., \& Lespier, Z. (2020). Desarrollo y validación de la Escala de Distancia Social hacia Personas Trans. Revista Evaluar, 20(1), 32-48. Recuperado de https://revistas.unc.edu.ar/index.php/revaluar
} 


\section{Introducción}

Las sociedades y las culturas actuales son, en su mayoría, cisnormalistas (American Psychological Association [APA], 2015; Coleman et al., 2012; Ramos-Pibernus, Rodríguez-Madera, Ramos, Padilla, \& Varas-Díaz, 2016). Esto implica que existen relaciones sociopolíticas de poder que normalizan y reglamentan el género y el sexo biológico de cada persona. Se tiene una visión dicótoma inflexible, en donde se ignora completamente la diversidad y se asume que toda persona es cisgénero y cisexual. Es decir, que toda persona está conforme con los constructos sociales del género que se le inculcó por su sexo biológico y que toda persona está conforme con los genitales y características sexuales con los que nació (Asociación de Psicología de Puerto Rico [APPR], 2014; Rodríguez-Madera, 2012; LGBT Advisory Comittee \& San Francisco Human Right Commission, 2011).

A consecuencia de estas actitudes y creencias negativas, se producen culturas transnegativistas. En estas culturas se crea un estigma que culmina en el repudio, prejuicio y discriminación hacia aquellas personas cuyo sexo y/o su género no está conforme con los constructos polarizados de hombre-mujer o masculino-femenino (APA, 2015; APPR, 2014; Ramos-Pibernus et al., 2016). La cultura crea entonces una división en la cual se observa a las personas diversas en sus identidades de sexo y de género como «los otros». Los otros pasan a ser un grupo de personas que, dado que sus identidades no se alinean con lo socialmente establecido, comienzan a adquirir características usualmente negativas y estereotipos que se convierten en esquemas conflictivos para muchas personas. Estos esquemas crean una intolerancia a todo hombre con masculinidades diversas y a aquel que traspase a cualquier nivel de la feminidad. Igualmente, a toda mujer con feminidades diversas y a toda aquella que traspase a lo masculino. No obstante, este ideal y estos límites de lo femenino y lo masculino son mayormente ambiguos $\mathrm{y}$, como todo constructo social, cambian de acuerdo a la cultura, el tiempo y el contexto (APA, 2012).

A pesar de tal panorama, los humanos son sumamente diversos y todos en su mayoría transgreden los constructos del género de alguna u otra manera. Como ejemplo de esto podemos mencionar instancias que van desde el hombre que cocina, hasta la mujer que trabaja en construcción. Sin embargo, a pesar de que estamos conscientes de la señalada transgresión, la sociedad define los límites y estos están altamente influenciados por controles sociopolíticos, ideales religiosos y posturas conservadoras (Francia-Martínez, Esteban, \& Lespier, 2017; Horn, 2013). Por lo tanto, solo se ven perjudicadas aquellas personas que no están dentro de esos límites socialmente establecidos (APPR, 2014; Rodríguez-Madera, 2009).

El panorama es más nocivo y perjudicial cuando hablamos de las personas cuya «transgresión» no pretende ser pasajera, sino que de cierta manera deciden renunciar a los constructos establecidos para transitarlos, combinarlos o simplemente alejarse de ellos. Este es el caso de la comunidad trans, una comunidad compuesta por personas no-cisgénero y/o no-cisexuales. En otras palabras, personas que no se encuentran conformes con el género que se les asignó al nacer y/o que manifiestan inconformidad parcial o total con el sexo de nacimiento. Este grupo de personas son mejor conocidas como personas trans, término que conglomera a las personas transgénero, transexuales, andróginas, agénero, bigénero, entre otras (APA, 2015; APPR, 2014).

Investigaciones recientes han encontrado que las personas trans han sido mucho más estigmatizadas, discriminadas y marginadas que las personas gays, lesbianas y bisexuales. De hecho, 
estudios han encontrado que la comunidad trans ha sido también la más invisibilizada y menos estudiada a nivel científico dentro la comunidad de personas lesbianas, gays, bisexuales y trans $(\mathrm{Co}-$ leman et al., 2012; McCann \& Sharek, 2014; Rodríguez-Madera, 2012). Este dato es alarmante, sobre todo si tomamos en consideración que dada la naturaleza de sus necesidades físicas y psicológicas son un sector que amerita atención multidisciplinaria. Se ha encontrado que esta comunidad presenta múltiples determinantes sociales que impactan su salud, especialmente en lo que a su salud mental se refiere (Rodríguez-Madera et al., 2016). Francia-Martínez et al. (2017), encontraron en una muestra de psicoterapeutas que, aunque presentaron en promedio actitudes positivas hacia la comunidad trans, informaron a su vez un grado moderado de distancia social. Es decir, por un lado informan no tener actitudes negativas hacia esta población, pero por otro lado informan que no vivirían con una persona trans bajo el mismo techo. Es en estas medidas incongruentes en donde mejor se puede observar el prejuicio consciente versus el inconsciente.

\section{Marco teórico}

Según Wark y Galliher (2007) Robert Park presentó a Emory Bogardus el concepto de distancia social, lo cual lo inspiró a construir la primera la escala que medía distancia social en 1926 con la discriminación por raza como motivo principal. Históricamente, el término distancia social se ha utilizado para reducir a categorías medibles los grados de comprensión e intimidad que caracterizan las relaciones personales y sociales en general (Wark \& Galliher, 2007). También, se ha definido como el grado de intimidad y comprensión que existe entre individuos o grupos sociales (Hughes, Johnson, Masuoka, Redfield, $\&$ Wirth, 1950). En este sentido, se puede definir distancia social como una medida de separación social entre grupos causada por diferencias reales o percibidas entre grupos de personas; estas diferencias suelen ser categorías socialmente establecidas por grupos mayoritarios que suelen percibirse como la norma. Consistentemente, la literatura científica vincula la distancia social con el prejuicio, entendiendo este último como la disposición más o menos instintiva y espontánea a mantener distancias sociales con otros grupos o individuos con ciertas características o atributos (Choi, Lee, Lee, \& Kim, 2017; Maurer \& Keim, 2018; Wark \& Galliher, 2007; Weaver, 2008).

La distancia social es además un trecho que se forma entre dos grupos por sus diferencias, usualmente marcado por estigma, estereotipos y/o prejuicios. Esta distancia entre los grupos produce una falta de contacto suficiente o prolongado entre estos. Esto propicia el desarrollo de la separación de los mismos, pudiendo culminar en actos de violencia y de agresión, tales como microagresiones, discriminación, marginación, violencia sexual, entre otros. Por lo tanto, la distancia social es una variable de medición de los vínculos de unión o segregación de grupos sociales y mide el conflicto que se genera en estas relaciones (Arteaga-Botello \& Lara-Carmona, 2004).

Según Navalles-Gómez (2011, p. 173), "la distancia y la cercanía se develan como los extremos humanos de la empatía”, implicando que cuando existe distancia hacia algún grupo se recrea un espacio de pugna, recalcando un ideal, un discurso, un sentimiento que, al final, expone una distinción. Existe evidencia de que se puede tener un bajo nivel de prejuicio hacia algún grupo en particular $\mathrm{y}$, no obstante, mostrar una conducta prejuiciada (Devine, Monteith, Zuwerink, \& Elliot, 1991), creando así una disonancia cognitiva entre cognición-conducta. Por ejemplo, 
mostrar expresiones favorables hacia las personas LGB en lo cotidiano, pero hacer chistes ridiculizando la homosexualidad en el lugar de trabajo. Según Fernández-Rodríguez y Calderón-Squiabro (2014, p. 53), “...parece haber una discrepancia en lo que se piensa y cómo se actúa cuando se trata de los prejuicios enraizados social y culturalmente".

En Puerto Rico, el constructo de distancia social se ha utilizado mayormente para medir distancia con respecto a minorías sexuales y de género. García (1984), comparó los prejuicios y la distancia social hacia los hombres gay y las mujeres lesbianas entre diferentes facultades universitarias, y encontró distancia social en todas ellas, pero más distancia en la facultad de Ciencias Naturales. Estos resultados implican que la distancia social hacia una comunidad específica podría depender del tipo de educación que recibe la persona o del área de estudio en que se desempeña. Toro-Alfonso y Varas-Díaz (2004), Fernández-Rodríguez y Calderón-Squiabro (2014), y Nieves-Rosa (2012) repitieron este estudio en diversas universidades públicas del país y encontraron resultados similares. Además, encontraron que esta distancia era mucho mayor en los hombres y menor en aquellas personas que conocían a una persona gay o lesbiana.

Desde la disciplina de la psicología, Vázquez-Rivera, Nazario-Serrano y Sayers-Montalvo (2012) y Vázquez-Rivera, Esteban y Toro-Alfonso (2018) encontraron algunas actitudes negativas y niveles moderados de distancia social por parte de profesionales de la psicología clínica y estudiantes de dicha disciplina. Francia-Martínez et al. (2017), encontraron también algunas actitudes negativas y distancia social moderada por parte de estudiantes y profesionales de psicología hacia la comunidad trans. A su vez, Rodríguez-Polo et al. (2018) hallaron bajos niveles de actitudes negativas y baja distancia social por parte de per- sonas empleadas en la Isla hacia personas gay y lesbianas. Por otro lado, desde la disciplina de la Salud Pública, González-Guzman (1998), también midió actitudes de prejuicio y distancia social hacia personas gays y lesbianas, y encontró mayores niveles de distancia social en estudiantes de segundo y tercer año de dicha concentración académica.

\section{Propósito del estudio}

En la actualidad no existen escalas para medir la distancia social hacia las personas trans. La variable de distancia social fue utilizada inicialmente para medir la distancia hacia personas con trastornos mentales (Grandón-Fernández, Cova-Solar, Saldivia-Bórquez, \& Bustos-Navarrete, 2015; Link, Cullen, Frank, \& Wozniak, 1987). Recientemente, se ha utilizado para medir la distancia hacia personas gays y lesbianas (Coppari et al., 2014; Fernández-Rodríguez \& Calderón-Squiabro, 2014; García, 1984; Rodríguez-Polo et al., 2018; Toro-Alfonso \& Varas-Díaz, 2004; Vázquez-Rivera et al., 2018). Tomando esto en consideración, el presente trabajo tuvo como propósito desarrollar y validar una escala para medir distancia social hacia la comunidad trans en una muestra de profesionales y estudiantes graduados de psicología. Con este fin, se examinó la validez de constructo del instrumento y se evaluó la confiabilidad del instrumento para corroborar si los ítems son consistentes en la medida del constructo distancia social hacia las personas trans. Como objetivo secundario, se evaluó si existían diferencias en la distancia social entre estudiantes y profesionales de la psicología. 


\section{Método}

\section{Diseño de investigación}

Esta investigación fue de tipo cuantitativo no-experimental, de alcance exploratorio, con un diseño de estudio instrumental (Ato, López-García, \& Benavente, 2013; Montero \& León, 2007). En ella se desarrolló y validó preliminarmente la Escala de Distancia Social hacia Personas Trans (DS-T) con una muestra de profesionales y estudiantes de psicología.

\section{Participantes}

Se trabajó con una muestra no probabilística constituida por 127 participantes (68 profesionales de la psicología y 59 estudiantes graduados de psicología), que decidieron confidencial y voluntariamente participar del estudio. La edad promedio de la muestra fue de 37.17 años (DE = 12.23). En la Tabla 1 se desglosa el resto de los datos sociodemográficos de los participantes. Los criterios de inclusión para ambos grupos fueron: (a) ser mayor de 21 años de edad y (b) tener experiencia o práctica supervisada en intervenciones de psicoterapia y/o evaluaciones psicológicas.

\section{Instrumentos}

Cuestionario de datos generales. Para identificar las características sociodemográficas de la muestra, se desarrolló un cuestionario de datos generales que recogía información importante en cuanto a la edad, el sexo, el género, el estado civil, la orientación sexual y la afiliación religiosa.

\section{Escala de Distancia Social hacia Personas Trans} $(\boldsymbol{D S}-\boldsymbol{T})$. Este instrumento fue construido por los
Tabla 1

Datos sociodemográficos de la muestra.

\begin{tabular}{lcc}
\hline $\begin{array}{l}\text { Variable } \\
\text { Sexo }\end{array}$ & $\boldsymbol{f}$ & $\mathbf{\%}$ \\
\hline Hombre & 40 & 31.5 \\
Mujer & 87 & 68.5 \\
& & \\
Género & & \\
\hline Masculino & 40 & 31.5 \\
Femenino & 86 & 67.7 \\
Queer & 1 & 0.8
\end{tabular}

Orientación sexual

\begin{tabular}{lcc}
\hline Heterosexual & 100 & 78.7 \\
Homosexual & 19 & 15.0 \\
Bisexual & 5 & 3.9 \\
Pansexual & 1 & 0.8 \\
Valores perdidos & 2 & 1.6
\end{tabular}

Estado civil

$\begin{array}{lcc}\text { Solteros } & 45 & 35.4 \\ \text { Casados } & 33 & 26.0 \\ \text { Convivencia } & 18 & 14.2 \\ \text { Divorciados } & 16 & 12.6 \\ \text { Noviazgo } & 13 & 10.2 \\ \text { Viudos } & 2 & 1.6\end{array}$

\begin{tabular}{lcc} 
Religión & & \\
\hline Católica & 59 & 46.5 \\
Protestante & 6 & 4.7 \\
Evangélica & 13 & 10.2 \\
Otra & 12 & 9.4 \\
Ninguna & 35 & 27.6 \\
Valores perdidos & 2 & 1.6
\end{tabular}

Rama en la psicología

\begin{tabular}{lcc} 
Psicología clínica & 46 & 36.2 \\
Consejería psicológica & 18 & 14.2 \\
Psicología escolar & 4 & 3.1 \\
Estudiante psicología clínica & 52 & 40.9 \\
Estudiante consejería psicológica & 6 & 4.7 \\
Estudiante psicología escolar & 1 & 0.8 \\
\hline
\end{tabular}

Nota. $(\mathrm{N}=127)$ 
investigadores con el propósito de desarrollar una justa medida que examine el distanciamiento social hacia personas trans. Operacionalmente, la distancia social hacia personas trans se define como la distancia que una persona indica que existe entre ella y las personas trans, mediante el respaldo de ciertas declaraciones (en este caso, los ítems de la DS-T). Con este propósito se desarrollaron 13 ítems que recogen varios aspectos de la comunidad trans en general. Los ítems fueron sometidos a la opinión de 10 jueces conocedores y estudiosos de la comunidad LGBT en Puerto Rico con el objetivo de identificar si los ítems del instrumento son pertinentes al distanciamiento social hacia personas trans (método de Lawshe). Para rechazar o retener los ítems se utilizó el content validity ratio (CVR). Para interpretar los resultados, utilizamos los valores críticos recalculados por Wilson, Pan y Schumsky (2012). De acuerdo con estos autores, el valor mínimo requerido para 10 jueces, según la tabla de Schipper, es .62 para aceptar un ítem como esencial. Todos los ítems obtuvieron valores aceptables, por lo cual permanecieron en el instrumento. La escala se responde seleccionando la alternativa de preferencia en cuanto a la distancia social: 1 (Viviría con ellos/as bajo la misma habitación), 2 (Viviría con ellos/as bajo el mismo techo), 3 (Los/as aceptaría como amigos/as), 4 (Preferiría tenerlos/as como conocidos/as y no como amigos/as), 5 (Los/ as excluiría de mis relaciones sociales), y 6 (No quisiera ni verles).

\section{Procedimientos}

La investigación fue aprobada por el Institutional Review Board (IRB) de la Universidad Carlos Albizu de Puerto Rico (Fall-14-09). Luego se procedió a recopilar la información de manera presencial y en línea, a través del portal surveymonkey.com. Se utilizó una hoja de consentimiento informado, donde no era necesario identificarse con nombre, para notificar a los participantes: el propósito del estudio, la naturaleza voluntaria del estudio, los posibles riesgos y beneficios, así como su derecho a retirarse del estudio en cualquier momento. Además, se informó sobre la duración de su participación y su derecho a obtener los resultados del estudio. Los acercamientos presenciales se realizaron mediante visitas a centros de trabajo de profesionales de la psicología, así como visitas a centros universitarios donde cursan estudiantes graduados de psicología.

\section{Estrategia de análisis}

Una vez recopilados los datos, analizamos los mismos utilizando el sistema para análisis estadísticos IBM SPSS Statistics (versión 23.0; IBM Corp., 2014). Específicamente, realizamos análisis descriptivos de la muestra (medias y desviaciones típicas), análisis de los ítems para conocer su índice de discriminación, análisis de factores y análisis de confiabilidad para calcular el coeficiente alfa de Cronbach y división en mitades de Spearman-Brown. Se realizó un análisis de comparación de medias prueba $t$ con el propósito de evaluar si existen diferencias estadísticamente significativas entre el estudiantado y los profesionales de la psicología.

Para el análisis factorial exploratorio se utilizó el método de extracción de mínimos cuadrados no ponderados (unweighted least squares) con rotación oblicua para identificar las variables latentes que subyacen en los ítems. Se utilizó este procedimiento de ajuste por dos razones principales: (1) el método de extracción de mínimos cuadrados no ponderados es el más recomendado 
actualmente para trabajar con muestras pequeñas, especialmente si el número de factores a retener es pequeño (Jung, 2013) y evita la aparición de casos Heywood (saturaciones mayores que la unidad y varianzas de error negativas), más frecuentes con otros métodos de estimación (Lloret-Segura, Ferreres-Traver, Hernández-Baeza, \& Tomás-Marco, 2014); y (2) la rotación oblicua es más precisa y provee más información que la rotación octogonal (Schmitt, 2011). Como criterios de aceptación, consideramos aquellos ítems con una carga factorial mayor a .50 en un solo factor (Stevens, 2002). Para conocer la capacidad de discriminación de los ítems de los factores se calculó el índice de correlación ítem-total $\left(r_{\text {bis }}\right)$. Para conocer el grado de confiabilidad de la escala, se calcularon el coeficiente alfa de Cronbach, alfa de Cronbach estandarizado y el coeficiente de división en mitades de Spearman-Brown. Field (2013) y Kline (2000) indican que índices mayores a .70 son aceptables.

\section{Resultados}

Análisis descriptivos

Con la intención de conocer la distancia social de los profesionales y estudiantes de psicología hacia personas trans, se organizaron descriptivamente (por frecuencia) las respuestas de cada uno de los ítems de la DS-T (véase Tabla 2). Los datos muestran que casi la mitad de profesionales y estudiantes de psicología aceptarían como amistades a personas trans. No obstante, no vivirían bajo la misma habitación ni vivirían bajo el mismo techo con personas que de alguna manera transgreden el género o el sexo. Es decir, no presentan cercanía ni aceptación incondicional. Ninguno de los participantes respondió que no quisieran ni verlos/as, y solo una persona señaló que los excluiría de sus relaciones sociales.

Análisis factorial exploratorio

Para determinar la validez de construcción lógica del instrumento, llevamos a cabo un análisis de factores utilizando el método de extracción de mínimos cuadrados no ponderados con rotación oblicua. Los resultados mostraron una estructura unifactorial que explicaba el 92\% de la varianza de los datos originales. Todos los ítems de la escala original cumplieron con cargas factoriales adecuadas (ver Tabla 3), por lo cual cumplieron con los criterios de aceptación detallados anteriormente. Las pruebas de Kaiser-Meyer-Olkin $(\mathrm{KMO}=.903)$ y la prueba de esfericidad Bartlett $\left(\chi_{(78)}^{2}=4026.526, p<.001\right)$ apoyaron la adecuación de los datos de muestreo para el análisis.

\section{Análisis de discriminación de items}

Con los datos obtenidos se llevó a cabo un análisis de los ítems para determinar sus índices de discriminación. Los resultados mostraron unos índices de discriminación que fluctuaban de .94 a .98. Al observar los valores obtenidos, identificamos que todos los ítems obtuvieron índices de discriminación dentro de los puntos de corte $\left(r_{\text {bis }}\right.$ $>$.30) sugeridos en la literatura (Field, 2013; Kline, 2000). En la Tabla 3 presentamos los índices de discriminación por ítem.

\section{Análisis de confiabilidad}

Los 13 ítems fueron sometidos a un análisis de consistencia interna para determinar el 
índice de confiabilidad de la escala. Para ello utilizamos dos métodos: el coeficiente alfa de Cronbach y división en mitades de Spearman-Brown. Los resultados mostraron un coeficiente alfa de .99 y de .98 para la prueba Spearman-Brown. En la Tabla 4 se presentan el alfa de Cronbach, el coeficiente de la prueba Spearman-Brown, la media y la desviación estándar de la DS-T.

\section{Análisis de comparación}

Se realizó un análisis de comparación de medias prueba $t$ con el propósito de evaluar si existen diferencias estadísticamente significativas entre las medias de distancia social hacia personas trans entre profesionales de la psicología y estudiantes graduados de psicología (ver Tabla 5). Los análisis reflejaron que no existen diferencias estadísticamente significativas en distancia social hacia personas trans entre profesionales de la psicología y estudiantes graduados de psicología.

\section{Discusión}

En Puerto Rico, así como en muchos países del mundo, la comunidad trans es víctima de distanciamiento social, discriminación, prejuicio y rechazo en todos los niveles. No obstante, la comunidad científica del país no contaba con un instrumento diseñado para medir este fenómeno en la Isla. De hecho, como se ha evidenciado, no existe un instrumento en español que mida este constructo. Es por ello que el presente estudio tuvo como propósito desarrollar, validar y examinar las propiedades psicométricas de la Escala de Distancia Social hacia las Personas Trans (DS-T) con una muestra de profesionales y estudiantes graduados de psicología. Los resultados obtenidos confirman que el instrumento posee las propiedades psicométricas adecuadas para medir distanciamiento social hacia las personas trans.

En términos teóricos, el análisis factorial exploratorio reveló la existencia de un solo factor latente en los ítems del instrumento. Esto evidencia cómo el prejuicio y la distancia social hacia la comunidad trans son consistentes en todas sus expresiones y formas; a tal grado que casi la mitad de los participantes, a pesar de estar vinculados con la profesión de la psicología, cuyo trabajo es de una naturaleza que requiere niveles bajos de distancia social, no presentan cercanía ni aceptación incondicional hacia esta comunidad. Por esta razón, mantener un discurso académico y profesional de inclusión y tener un perfil de no prejuicio no asegura cercanía social hacia la comunidad trans (Devine et al., 1991).

Por otra parte, no se observaron diferencias estadísticamente significativas en la distancia social entre estudiantes y profesionales de la psicología. Esto podría implicar, entre otras cosas, que la distancia social hacia las personas trans no se debe a un asunto generacional. También nos provee evidencia de que la experiencia en la profesión de la psicología no disminuye el distanciamiento hacia una comunidad marginada. Según Navalles-Gómez (2011), la distancia y la cercanía son los extremos de la empatía, por lo que mientras mayor es la distancia hacia una persona o comunidad, menor será la probabilidad de empatía. La empatía es una destreza básica y esencial que debe tener toda aquella persona que provee ayuda, especialmente aquellas que proveen servicios de salud mental. Un servicio sin empatía es posiblemente un servicio que no aporta a la salud, el bienestar y la calidad de vida de la persona que lo necesita. Este dato cobra aún más relevancia e importancia en el caso de la comunidad trans que, como se ha indicado previamente en este escrito, representa un grupo vulnerable de clientes 
Tabla 2

Frecuencias de las respuestas de los participantes en la DS-T.

\begin{tabular}{|c|c|c|c|c|c|c|}
\hline & $\begin{array}{c}\text { Viviría con } \\
\text { ellos/as bajo } \\
\text { la misma } \\
\text { habitación }\end{array}$ & $\begin{array}{c}\text { Viviría } \\
\text { con ellos/ } \\
\text { as bajo } \\
\text { el mismo } \\
\text { techo }\end{array}$ & $\begin{array}{c}\text { Los/as } \\
\text { aceptaría } \\
\text { como } \\
\text { amigos/as }\end{array}$ & $\begin{array}{c}\text { Preferiría } \\
\text { tenerlos/ } \\
\text { as como } \\
\text { conocidos/as } \\
\text { y no como } \\
\text { amigos/as }\end{array}$ & $\begin{array}{l}\text { Los/as } \\
\text { excluiría de } \\
\text { mis relacio- } \\
\text { nes sociales }\end{array}$ & $\begin{array}{l}\text { No quisiera } \\
\text { ni verlos/as }\end{array}$ \\
\hline Hombres que se visten de mujer & $18(14 \%)$ & $48(38 \%)$ & $53(42 \%)$ & $8(6 \%)$ & $0(0 \%)$ & $0(0 \%)$ \\
\hline Mujeres que se visten de hombre & $21(17 \%)$ & $45(35 \%)$ & $52(41 \%)$ & $9(7 \%)$ & $0(0 \%)$ & $0(0 \%)$ \\
\hline $\begin{array}{l}\text { Una mujer transexual en hormonas } \\
\text { feminizantes }\end{array}$ & $21(17 \%)$ & $47(37 \%)$ & $51(40 \%)$ & $7(5 \%)$ & $1(1 \%)$ & $0(0 \%)$ \\
\hline $\begin{array}{l}\text { Un hombre transexual en hormonas } \\
\text { masculinizantes }\end{array}$ & $21(17 \%)$ & $48(38 \%)$ & $48(38 \%)$ & $9(7 \%)$ & $1(1 \%)$ & $0(0 \%)$ \\
\hline $\begin{array}{l}\text { Una mujer transexual en procesos qui- } \\
\text { rúrgicos corporales }\end{array}$ & $20(16 \%)$ & $44(35 \%)$ & $56(44 \%)$ & $6(5 \%)$ & $1(1 \%)$ & $0(0 \%)$ \\
\hline $\begin{array}{l}\text { Un hombre transexual en procesos } \\
\text { quirúrgicos corporales }\end{array}$ & $22(17 \%)$ & $43(34 \%)$ & $52(41 \%)$ & $9(7 \%)$ & $1(1 \%)$ & $0(0 \%)$ \\
\hline $\begin{array}{l}\text { Una mujer transexual que posea sus } \\
\text { genitales de nacimiento }\end{array}$ & $22(17 \%)$ & $48(38 \%)$ & $50(39 \%)$ & $6(5 \%)$ & $1(1 \%)$ & $0(0 \%)$ \\
\hline $\begin{array}{l}\text { Un hombre transexual que posea sus } \\
\text { genitales de nacimiento }\end{array}$ & $22(17 \%)$ & $45(35 \%)$ & $52(41 \%)$ & $6(5 \%)$ & $1(1 \%)$ & $0(0 \%)$ \\
\hline $\begin{array}{l}\text { Una mujer transexual o transgénero que } \\
\text { todavía parezca hombre físicamente }\end{array}$ & $19(15 \%)$ & $46(36 \%)$ & $55(43 \%)$ & $6(5 \%)$ & $1(1 \%)$ & $0(0 \%)$ \\
\hline $\begin{array}{l}\text { Un hombre transexual o transgénero que } \\
\text { todavía parezca mujer físicamente }\end{array}$ & $20(16 \%)$ & $45(35 \%)$ & $55(43 \%)$ & $6(5 \%)$ & $1(1 \%)$ & $0(0 \%)$ \\
\hline $\begin{array}{l}\text { Una persona que no parezca ni hombre } \\
\text { ni mujer (andrógina) }\end{array}$ & $18(14 \%)$ & $51(40 \%)$ & $49(39 \%)$ & $8(6 \%)$ & $1(1 \%)$ & $0(0 \%)$ \\
\hline $\begin{array}{l}\text { Una mujer que su expresión de género } \\
\text { sea masculina }\end{array}$ & $22(17 \%)$ & $46(36 \%)$ & $51(40 \%)$ & $7(5 \%)$ & $1(1 \%)$ & $0(0 \%)$ \\
\hline $\begin{array}{l}\text { Un hombre que su expresión de género } \\
\text { sea femenina }\end{array}$ & $22(17 \%)$ & $47(37 \%)$ & $52(41 \%)$ & $6(5 \%)$ & $0(0 \%)$ & $0(0 \%)$ \\
\hline
\end{tabular}

con necesidades de tratamiento únicas y específicas. La empatía es un elemento clave para poder establecer una relación terapéutica efectiva, no obstante, se ha reportado en la literatura falta de competencias clínicas y actitudes de prejuicio y rechazo dentro de la misma profesión de psicología (Chang \& Singh, 2016; Francia-Martínez et al., 2017; Sue \& Sue, 2008). Por lo tanto, un instrumento que nos brinda información sobre la distancia social que tiene un profesional hacia una comunidad, paralelamente nos brinda información sobre la empatía que tendrá con esta población en el ejercicio de su profesión.
En cuanto a la confiabilidad de la escala, se obtuvieron índices superiores al mínimo recomendado por la literatura científica (Field, 2013; Kline, 2000), lo cual sugiere que la DS-T es un instrumento estable, reproducible y consistente en la medida de distanciamiento social hacia las personas trans. Asimismo, las correlaciones de cada ítem con la puntuación total manifiestan una consistencia interna notable. Esto sugiere que los ítems de la versión final discriminan adecuadamente y son capaces de diferenciar personas con distintos niveles de distancia social hacia la comunidad trans. 
Tabla 3

Cargas factoriales de los ítems y sus índices de discriminación.

\begin{tabular}{llcc}
\hline & Ítems & Factor & $\boldsymbol{r}_{\text {bis }}$ \\
\hline 1 & Hombres que se visten de mujer & .944 & .94 \\
2 & Mujeres que se visten de hombre & .961 & .96 \\
3 & Una mujer transexual en hormonas feminizantes & .956 & .94 \\
4 & Un hombre transexual en hormonas masculinizantes & .968 & .95 \\
5 & Una mujer transexual en procesos quirúrgicos corporales & .959 \\
6 & Un hombre transexual en procesos quirúrgicos corporales & .960 \\
7 & Una mujer transexual que posea sus genitales de nacimiento & .962 \\
8 & Un hombre transexual que posea sus genitales de nacimiento & .979 \\
9 & Una mujer transexual o transgénero que todavía parezca hombre físicamente & .96 \\
10 & Un hombre transexual o transgénero que todavía parezca mujer físicamente & .979 \\
11 & Una persona que no parezca ni hombre ni mujer (andrógina) & .96 \\
12 & Una mujer que su expresión de género sea masculina & .95 \\
13 & Un hombre que su expresión de género sea femenina. & .95 \\
\hline
\end{tabular}

Nota. Factor $=$ carga factorial obtenida por el ítem en el análisis factorial; $r_{\text {bis }}=$ índice de discriminación. $(\mathrm{N}=127)$.

Tabla 4

Confiabilidad y estadística descriptiva de la DS-T.

\begin{tabular}{ccccccc}
\hline Escala & Ítems & Alfa & Alfa $_{\text {est }}$ & Spearman Brown & M & DE \\
\hline DS-T & 13 & .99 & .99 & .98 & 30.97 & 10.48 \\
\hline
\end{tabular}

Nota. Alfa $_{\text {est }}=$ Alfa estandarizado; $\mathrm{M}=$ media; $\mathrm{DE}=$ desviación estándar. $(\mathrm{N}=127)$.

Tabla 5

Resultados del análisis de comparación de medias prueba $t$ y tamaño del efecto.

\begin{tabular}{cccccccc}
\hline Distancia Social & $\mathbf{N}$ & $\mathbf{M}$ & $\mathbf{D E}$ & $\boldsymbol{t}$ & $\boldsymbol{d f}$ & $\boldsymbol{p}$ & $\boldsymbol{d}$ \\
& & & & & & \\
& & & & & \\
Profesionales & 67 & 31.43 & 10.58 & .531 & 123 & .60 & .10 \\
Estudiantes & 58 & 30.43 & 10.42 & & & \\
\hline
\end{tabular}

Nota. $\mathrm{N}=$ participantes; $\mathrm{M}=$ medias; $g l=$ grados de libertad; $\mathrm{DE}=$ desviación estándar; $t=$ valor de la prueba $t ; p=$ significancia; $d=$ tamaño del efecto $d$ de Cohen. $(\mathrm{N}=127)$.

En cuanto a las implicaciones prácticas, se demostró que la DS-T puede ser utilizada para el desarrollo de nuevas investigaciones en el campo de la psicología caribeña. Este es un gran avance, dado que en Latinoamérica no existía un instrumento que examine el distanciamiento social hacia la comunidad trans. Aunque el concepto de distancia social hacia la comunidad gay y lesbiana ha sido estudiado y replicado, el concepto no se había adaptado a la comunidad trans; comunidad sobre la que recae un mayor nivel de prejuicio por parte de la sociedad en general e inclusive por parte de la misma comunidad LGBT+. Del mismo modo, el concepto de distancia social se 
puede adaptar a cualquier comunidad desaventajada tales como las que incluyen personas con necesidades especiales, diversidad funcional, usuarias de sustancias, diversidad étnica, con VIH/ SIDA, entre otras. Conocer el grado de cercanía o de distancia social hacia estos grupos permitiría trabajar en recomendaciones y planes de acción para la reducción y/o eliminación de esta barrera terapéutica.

En términos de corrección, las puntuaciones de la DS-T se calculan mediante la sumatoria de los 13 ítems para obtener un índice general. Para obtener la puntuación total de la escala se deben sumar las puntuaciones alcanzadas en cada ítem (no hay ítems inversos). Al finalizar la sumatoria se clasifican las puntuaciones de acuerdo a los cuartiles: baja o ninguna distancia (13-26), distancia moderada baja (31-45), distancia moderada alta (46-62) y distancia alta (63-78).

\section{Limitaciones y recomendaciones}

Varias limitaciones fueron identificadas en este estudio. Una de las limitaciones principales fue el tamaño de la muestra, la cual fue pequeña para un estudio instrumental de esta envergadura, considerando la cantidad de variables en el mismo. No obstante, se tomó en consideración el tamaño de la muestra y el nivel de significancia al momento de tomar decisiones sobre los ítems, así como un método de extracción adecuado a dicha limitación (Jung, 2013). Esto permite sostener la calidad y confiabilidad de los resultados obtenidos (Stevens, 2002). Cabe añadir que los análisis se llevaron a cabo con una sola muestra, lo cual no es recomendable, ya que se pueden repetir los mismos errores de muestreo. Otra limitación fue que no se logró establecer la confiabilidad del instrumento a través del tiempo, solo se pudo hacer a través de sus elementos.

Tomando en consideración las limitaciones identificadas, se recomienda realizar un nuevo estudio y administrar la DS-T a una muestra mayor de participantes, que incluya participantes de la población general, para examinar si se sostienen las propiedades psicométricas del instrumento y realizar el proceso de validación cruzada. De igual forma, se debe llevar a cabo un análisis de factores confirmatorio y examinar la confiabilidad temporal a través de la técnica de prueba-reprueba. Por último, se recomienda validar la DS-T en otras poblaciones latinoamericanas para examinar sus propiedades psicométricas en distintos contextos nacionales e internacionales.

En síntesis, la Escala de Distancia Social hacia las Personas Trans (DS-T) mostró preliminarmente indicadores adecuados de validez y confiabilidad, lo que sugiere que la DS-T tiene el potencial de contribuir al avance de la investigación con miras a comprender con mayor amplitud el fenómeno del distanciamiento social y el prejuicio hacia la comunidad trans. Se espera que la DS-T sea de beneficio en el ámbito de la investigación y facilite nuevos conocimientos sobre este fenómeno en Puerto Rico y en América Latina.

\section{Referencias}

American Psychological Association. (2012). Guidelines for psychological practice with lesbian, gay, and bisexual clients. American Psychologist, 67(1), 10-42. doi: 10.1037/a0024659

American Psychological Association. (2015). Guidelines for psychological practice with transgender and gender nonconforming people. American Psychologist, 70(9), 832-864. doi: 10.1037/a0039906

Arteaga-Botello, N., \& Lara-Carmona, V. L. (2004). Violencia y distancia social: Una revisión. Papeles de Población, 10(40), 169-191. Recuperado de: ht- 
tps://rppoblacion.uaemex.mx/index

Asociación de Psicología de Puerto Rico. (2014). Estándares para el trabajo e intervención en comunidades lesbianas, gay, bisexuales e identidades trans (LGBT). San Juan, Puerto Rico: Autor.

Ato, M., López-García, J. J., \& Benavente, A. (2013). Un sistema de clasificación de los diseños de investigación en psicología. Anales de Psicología, 29(3), 1038-1059. doi: 10.6018/analesps.29.3.178511

Chang, S. C., \& Singh, A. A. (2016). Affirming psychological practice with transgender and nonconforming people of color. Psychology of Sexual Orientation and Gender Diversity, 3(2), 140-147. doi: 10.1037/ $\operatorname{sgd} 0000153$

Choi, O., Lee, K., Lee, K., \& Kim, J. (2017). Influences of stereotype and social distance on prejudice toward African Americans. Journal of Psychology in Africa, 27(1), 13-17. doi: 10.1080/14330237.2016.1268262

Coleman, E., Bockting, W., Botzer, M., Cohen-Kettenis, P., DeCuypere, G., Feldman, J., ... Zucker, K. (2012). Standards of care for the health of Transsexual, transgender and gender-nonconforming people, Version 7. International Journal of Transgenderism, 13(4), 165-232. doi: $10.1080 / 15532739.2011 .700873$

Coppari, N., Arcondo, G., Bagnoli, L., Chaves, M., Corvalán, M., Enciso, A., ... Rodríguez, X. (2014). Prejuicio y distancia social hacia la homosexualidad en universitarios de psicología de Paraguay. Salud y Sociedad, 5(3), 240-252. doi: 10.22199/ s07187475.2014.0003.00001

Devine, P. G., Monteith, M. J., Zuwerink, J. R., \& Elliot, A. J. (1991). Prejudice with and without compunction. Journal of Personality \& Social Psychology, 60(6), 817-830. doi: 10.1037/0022-3514.60.6.817

Fernández-Rodríguez, M. C., \& Calderón-Squiabro, J. (2014). Prejuicio y distancia social hacia personas homosexuales por parte de jóvenes universitarios. Revista Puertorriqueña de Psicología, 25(1), 52-
60. Recuperado de http:/www.ojs.repsasppr.net/ index.php/reps/index

Field, A. P. (2013). Discovering statistics using SPSS. Londres, Inglaterra: SAGE.

Francia-Martínez, M., Esteban, C., \& Lespier, Z. (2017). Actitudes, conocimiento y distancia social de psicoterapeutas con la comunidad transgénero y transexual. Revista Puertorriqueña de Psicología, 28(1), 98-113. Recuperado de http://www.ojs.repsasppr.net/index.php/reps/index

García, H. (1984). Actitudes hacia homosexuales y las lesbianas de cuatro grupos de estudiantes universitarios (Tesis de maestría inédita). Universidad de Puerto Rico, Río Piedras, Puerto Rico.

González-Guzmán, M. (1998). Actitud de los estudiantes de salud pública general y educación en salud de la escuela graduada de salud pública de la Universidad de Puerto Rico, Recinto de Ciencias Médicas, hacia los homosexuales y lesbianas (Tesis de maestría inédita). Universidad de Puerto Rico, Recinto de Ciencias Médicas, Puerto Rico.

Grandón-Fernández, P., Cova-Solar, F., Saldivia-Bórquez, S., \& Bustos-Navarrete, C. (2015). Análisis psicométrico y adaptación de la Escala de Distancia Social (DS) en una muestra chilena. Salud Mental, 38(2), 117-122. doi: 10.17711/SM.01853325.2015 .016

Horn, S. (2013). Attitudes about sexual orientation. En C. Patterson \& A. D'Angelis (Eds.), Handbook of psychology and sexual orientation (pp. 239-251). New York, N.Y.: Oxford University. doi: 10.1093/ acprof:oso/9780199765218.003.0017

Hughes, E. C., Johnson, C. S., Masuoka, J., Redfield, R., \& Wirth, L. (1950). The collected papers of Robert E. Park. Glencoe, IL: The Free.

IBM Corp. (2014). IBM SPSS Statistics for Windows (version 23.0). [software de cómputo]. Armonk, NY: IBM Corp.

Jung, S. (2013). Exploratory factor analysis with small sample sizes: A comparison of three approaches. Be- 
havioural Processes, 97(1), 90-95. doi: 10.1016/j. beproc.2012.11.016

Kline, P. (2000). The Handbook of Psychometric Testing ( $2^{\mathrm{a}}$ ed.). New York, NY: Routledge.

LGBT Advisory Committee, \& San Francisco Human Rights Commission. (2011). Bisexual invisibility: Impacts and recommendations. San Francisco, CA: San Francisco Human Rights Commission. Recuperado de https://sf-hrc.org

Link, B. G., Cullen, F. T., Frank, J., \& Wozniak, J. F. (1987). The social rejection of former mental patients: Understanding why labels matter. American Journal of Sociology, 92(6), 1461-1500. doi: $10.1086 / 228672$

Lloret-Segura, S., Ferreres-Traver, A., Hernández-Baeza, A., \& Tomás-Marco, I. (2014). El análisis factorial exploratorio de los ítems: Una guía práctica, revisada y actualizada. Anales de Psicología, 30(3), 1151-1169. doi: 10.6018/analesps.30.3.199361

Maurer, T. W., \& Keim, C. (2018). Teaching about prejudice with a Bogardus Social Distance Scale activity: Replication and extension. International Journal for the Scholarship of Teaching and Learning, 12(1), 1-10. doi: 10.20429/ijsotl.2018.120107

McCann, E., \& Sharek, D. (2014). Survey of lesbian, gay, bisexual and transgender people's experiences of mental health services in Ireland. International Journal of Mental Health Nursing, 23(2), 118-127. doi: 10.1111/inm.12018

Montero, I., \& León, O. G. (2007). A guide for naming research studies in psychology. International Journal of Clinical and Health Psychology, 7(3), 847862. Recuperado de https://www.redalyc.org

Navalles-Gómez, J. (2011). Acercamientos a la distancia social. Athenea Digital: Revista de Pensamiento e Investigación Social, 11(2), 173-190. doi: 10.5565/ $\mathrm{rev} / \mathrm{athenead} / \mathrm{v} 11 \mathrm{n} 2.763$

Nieves-Rosa, L. E. (2012). Homofobia al estilo universitario. Revista Puertorriqueña de Psicología, 23(2), 62-76. Recuperado de http://www.ojs.repsasppr. net

Ramos-Pibernus, A. G., Rodríguez-Madera, S. L., Padilla, M., Varas-Díaz, N., \& Vargas-Molina, R. (2016). Intersections and evolution of 'Butch-trans' categories in Puerto Rico: Needs and barriers of an invisible population. Global Public Health, 11(7), 966-980. doi: 10.1080/17441692.2016.1180703

Rodríguez-Madera, S. L. (2009). Género Trans: Transitando por las zonas grises. San Juan, Puerto Rico: Terranova.

Rodríguez-Madera, S. L. (2012). Trans-acciones de la carne: Criminalización de mujeres trans que ejercen el trabajo sexual. En S. Serrano (Ed.), Registros criminológicos contemporáneos (pp. 107-150). San Juan, Puerto Rico: Situm.

Rodríguez-Madera, S., Ramos, A., Padilla, M., \& Varas-Díaz, N. (2016). Radiografías de las comunidades Trans en Puerto Rico: Visibilizando femineidades y masculinidades alternas. En M. Vázquez-Rivera, A. Martínez-Taboas, M. Francia-Martínez \& J. Toro-Alfonso (Eds.), LGBT 101: Una mirada introductoria al colectivo (pp. 315-342). Hato Rey, Puerto Rico: Publicaciones Puertorriqueñas.

Rodríguez-Polo, J., Ayvar, A., Dávila, A., Andino, P., Quiñones, C., Rodriguez, L., ... Pacheco, T. (2018). Prejuicio y distancia social hacia las personas gays y lesbianas en una muestra de empleados en Puerto Rico. Revista Griot, 11(1), 16-33. Recuperado de https://revistas.upr.edu/index.php/griot/index

Schmitt, T. A. (2011). Current methodological considerations in exploratory and confirmatory factor analysis. Journal of Psychoeducational Assessment, 29(4), 304-321. doi: 10.1177/0734282911406653

Stevens, J. P. (2002). Applied multivariate statistics for the social sciences (4 ${ }^{\mathrm{a}}$ ed.). Mahwah, NJ: Lawrence Erlbaum.

Sue, D., \& Sue, D. M. (2008). Foundations of counseling and psychotherapy: Evidence based practice for a diverse society. New York, NY: J. Wiley and Sons. 
Toro-Alfonso, J., \& Varas-Díaz, N. (2004). Los otros: Prejuicio y distancia social hacia hombres gay y lesbianas en una muestra de estudiantes de nivel universitario. International Journal of Clinical and Health Psychology, 4(3), 537-551. Recuperado de https://aepc.es/ijchp/busca.php

Vázquez-Rivera, M., Nazario-Serrano, J., \& Sayers-Montalvo, S. K. (2012). La deseabilidad social en estudiantes graduados/as de psicología clínica y psicólogos/as clínicos con licencia. Revista Puertorriqueña de Psicología, 23(2), 94-106. Recuperado de http://www.ojs.repsasppr.net

Vázquez-Rivera, M., Esteban, C., \& Toro-Alfonso, J. (2018). Hacia una psicología libre de paños tibios: Actitudes, prejuicio y distancia social de psicoterapeutas hacia gays y lesbianas. Perspectivas en Psicología: Revista de Psicología y Ciencia Afines, 15(1), 17-30. Recuperado de https://www.redalyc. org/revista.oa? $\mathrm{id}=4835 \&$ tipo $=$ coleccion

Wark, C., \& Galliher, J. F. (2007). Emory Bogardus and the origins of the Social Distance Scale. The American Sociologist, 38(4), 383-395. doi: 10.1007/s12108007-9023-9

Weaver, C. N. (2008). Social distance as a measure of prejudice among ethnic groups in the United States. Journal of Applied Social Psychology, 38(3), 779795. doi: 10.1111/j.1559-1816.2007.00326.x

Wilson, F. R., Pan, W., \& Schumsky, D. A. (2012). Recalculation of the critical values for Lawshe's content validity ratio. Measurement and Evaluation in Counseling and Development, 45(3), 197-210. doi: 10.1080/07481756.2017.1308227 
Instrucciones:

En la columna B de la tabla que se presenta más adelante se le proveen distintos niveles de relaciones sociales. Dejándose llevar por sus sentimientos más que por su razón, señale con una marca de cotejo $(\sqrt{ })$ en el encasillado correspondiente, el nivel de relación social que estaría en disposición a sostener con cada una de las personas que se describen en la columna A. Marque solamente una alternativa.
El siguiente ejemplo tomado de otro contexto le ayudará a entender cómo cumplimentar la tabla. Ejemplo:

Señale con una marca de cotejo $(\sqrt{ })$ el nivel de relación social que estaría en disposición a sostener con cada una de las nacionalidades que se presentan a continuación.

\section{Columna A}

Columna B

\begin{tabular}{|c|c|c|c|c|c|}
\hline $\begin{array}{c}\text { Viviría } \\
\text { bajo la } \\
\text { misma ha- } \\
\text { bitación }\end{array}$ & $\begin{array}{l}\text { Viviría con bajo } \\
\text { el mismo techo } \\
\text { (en diferentes } \\
\text { habitaciones) }\end{array}$ & $\begin{array}{c}\text { Les } \\
\text { aceptaría } \\
\text { como } \\
\text { amistades }\end{array}$ & $\begin{array}{c}\text { Preferiría } \\
\text { tenerles como } \\
\text { personas cono- } \\
\text { cidas y no como } \\
\text { amistades }\end{array}$ & $\begin{array}{l}\text { Les excluiría } \\
\text { de mis rela- } \\
\text { ciones sociales }\end{array}$ & $\begin{array}{l}\text { No quisiera ni } \\
\text { verles }\end{array}$ \\
\hline
\end{tabular}

\begin{tabular}{lllllll}
\hline 1. & Alemanes & $\square$ & $\square$ & $\square$ & $\sqrt{ }$ & $\square$ \\
\hline 2. & Canadienses & $\square$ & $\sqrt{ }$ & $\square$ & $\square$ & $\square$ \\
\hline 3. & Franceses & $\square$ & $\sqrt{ }$ & $\square$ & $\square$ & $\square$ \\
\hline
\end{tabular}

* Si prefieres tener a las personas de Alemania como personas conocidas, pero no como amistades deberás colocar una marca de cotejo $(\sqrt{ })$ del modo que aparece en la tabla, o sea, en el encasillado correspondiente a la fila "alemanes" y la columna "preferiría tenerles como personas conocidas y no como amistades". Si pudiese vivir con una persona de Canadá bajo el mismo techo, pero no bajo la misma habitación, deberá colocar una marca de cotejo $(\sqrt{ })$ en el encasillado correspondiente a "canadienses" y "viviría con bajo el mismo techo" tal y como se muestra en la tabla. De igual forma con las personas de Francia, según sea su forma de sentir.

\begin{tabular}{|c|c|c|c|c|c|c|}
\hline \multirow[t]{2}{*}{ Columna A } & \multicolumn{6}{|c|}{ Columna B } \\
\hline & $\begin{array}{l}\text { Viviría bajo la } \\
\text { misma habitación }\end{array}$ & $\begin{array}{l}\text { Viviría con bajo } \\
\text { el mismo techo } \\
\text { (en diferentes } \\
\text { habitaciones) } \\
\end{array}$ & $\begin{array}{l}\text { Les aceptaría } \\
\text { como amis- } \\
\text { tades }\end{array}$ & $\begin{array}{c}\text { Preferiría tenerles } \\
\text { como personas } \\
\text { conocidas y no como } \\
\text { amistades } \\
\end{array}$ & $\begin{array}{l}\text { Les excluiría de } \\
\text { mis relaciones } \\
\text { sociales }\end{array}$ & $\begin{array}{l}\text { No quisiera ni } \\
\text { verles }\end{array}$ \\
\hline $\begin{array}{l}\text { 1. Hombres que } \\
\text { se visten de } \\
\text { mujer }\end{array}$ & $\square$ & $\square$ & $\square$ & $\square$ & $\square$ & $\square$ \\
\hline $\begin{array}{l}\text { 2. Mujeres que } \\
\text { se visten de } \\
\text { hombre }\end{array}$ & $\square$ & $\square$ & $\square$ & $\square$ & $\square$ & $\square$ \\
\hline
\end{tabular}


3. Una mujer transexual en hormonas feminizantes

4. Un hombre transexual en hormonas masculinizantes

5. Una mujer transexual en procesos quirúrgicos corporales

6. Un hombre transexual en procesos quirúrgicos corporales

7. Una mujer transexual que posea sus genitales de nacimiento

8. Un hombre transexual que posea sus genitales de nacimiento

9. Una mujer transexual o transgénero que todavía parezca hombre físicamente

10. Un hombre transexual o transgénero que todavía parezca mujer físicamente

11. Una persona que no parezca ni hombre ni mujer (andrógina)

12. Una mujer que su expresión de género sea masculina 
13. Un hombre que su expresión de género sea femenina

Método de corrección sumaria

Puntuación regular: los ítems se puntúan de 1 (Viviría bajo la misma habitación) a 6 (No quisiera ni verles).

Puntuación inversa: no hay ítems inversos. 